Boise State University

ScholarWorks

$11-3-2004$

Direct Comparison Between Phase Locked Oscillator and Direct Resonance Oscillator in the Noncontact Atomic Force Microscopy Under Ultrahigh Vacuum

Byung I. Kim

Boise State University 


\title{
Direct comparison between phase locked oscillator and direct resonance oscillator in the noncontact atomic force microscopy under ultrahigh vacuum
}

\author{
Byung I. Kim ${ }^{\mathrm{a}}$ \\ Department of Chemistry, University of Houston, Houston, Texas 77204
}

(Received 2 December 2002; accepted 26 July 2004; published 3 November 2004)

\begin{abstract}
I have demonstrated the advantage of the phase locked oscillator (PLO) over the conventional direct resonance oscillator (DRO) in noncontact mode atomic force microscopy (AFM) under ultrahigh vacuum. Direct comparison between PLO and DRO has been made in terms of background noise level, temporal response, and stability. Compared to the DRO method without phase coherence, the experimental results show that the PLO method is more effective in reducing the noise level and enhancing the stability over all force regimes in UHV noncontact AFM. The noise reduction and stability enhancement in PLO indicate the important role of the phase coherent effect in improving the capability of noncontact imaging in UHV. (C) 2004 American Institute of Physics.
\end{abstract}

[DOI: 10.1063/1.1806998]

Noncontact mode atomic force microscopy (AFM) in ultrahigh vacuum (UHV) is a very attractive technique because it provides atomic resolution images on insulating surfaces as well as conducting surfaces. ${ }^{1-4}$ However, the technique is still difficult and precarious for most laboratories due to its complexity. This is mainly due to the extremely high quality $(Q)$ factor of cantilevers, inherent in vacuum. The technique requires an additional circuit that employs frequency modulation. ${ }^{5}$ Frequency modulation uses a selfoscillating feedback loop to measure the frequency shift that arises from tip-sample interactions. Spontaneous vibration of the cantilever induced by thermal excitation keeps track of its resonance frequency, forming a direct resonance oscillator (DRO). When the oscillating cantilever is brought near the sample surface, the interaction between the tip and the sample causes a resonance frequency shift, $\Delta f$, which is used as the probing signal. ${ }^{5}$

In addition to the resonance frequency shift, the phase signal also shifts and can be used as a probing signal. Indeed, a phase locked loop (PLL) has been successfully employed through the phase locked oscillator (PLO) technique for atomic resolution imaging, ${ }^{2,3,6}$ magnetic force microscopy, ${ }^{7}$ and measuring the adhesion and the energy dissipation on metallic surface in UHV. ${ }^{8,9}$ Furthermore, its application extends to high $Q$ systems such as a tuning fork in ambient condition to control feedback for near field optical microscopy and shear force microscopy. ${ }^{10}$ More recently, high resolution images of biological samples in liquid have been acquired by enhancing $Q$ by 3 to 4 orders of magnitude through active feedback using a phase locked oscillator. ${ }^{11}$

Although the effectiveness and importance of the PLO technique are well demonstrated in noncontact AFM, direct

\footnotetext{
${ }^{a)}$ Author to whom correspondence should be addressed; electronic mail: ByungKim@boisestate.edu; present address: Department of Physics, Boise State University, Boise, Idaho 83725.
}

comparison between DRO and PLO has rarely been made so far. Here I present their performances in terms of noise level, temporal response, and tip-sample interaction as a function of separation distance between tip and sample by implementing a versatile, inexpensive, and easily modifiable circuit to an existing commercial AFM system.

I use an OMICRON UHV STM/AFM head with an optical beam deflection detection system. For the noncontact mode measurement, the cantilever can be oscillated by a thin piezo attached underneath the cantilever stage. RHK electronics and software are utilized for data acquisition, control of the microscope head, and data analysis. Figure 1(a) shows schematic diagrams of PLO and DRO. The vertical component of the cantilever vibration is detected with a quad-cell position sensitive photodiode (PSD) in the optical beam deflection technique and used as the input signal of both oscillators. PLO consists of a detector, a phase shifter and a PLL while DRO has automatic gain control (AGC), a demodulator, and a phase shifter. The output of the phase shifter that follows PLL or AGC is used as an excitation signal of the cantilever for proper tracking of resonance frequency. The resonance frequency shift, $\Delta f$, is used as a signal for servofeedback in noncontact mode operation. I could make in situ comparison between PLO and DRO by toggling one to the other with multiple switches.

Figure 1(b) shows the detailed schematic block diagram of PLO. I used a simple integrated PLL circuit LM 565 (National Semiconductor) with a maximum operating frequency of $500 \mathrm{kHz} .{ }^{12}$ The analog multiplier, as a phase detector, generates the phase difference between the excitation and response. The loop filter generates time averaged dc output as the input of a voltage controlled oscillator (VCO) by eliminating the frequency-doubled component after phase detection. Since the input of the VCO becomes an accurate measure of the frequency, PLL is used as an oscillator as well as a demodulator. The center frequency of the VCO, $f_{c}$, 

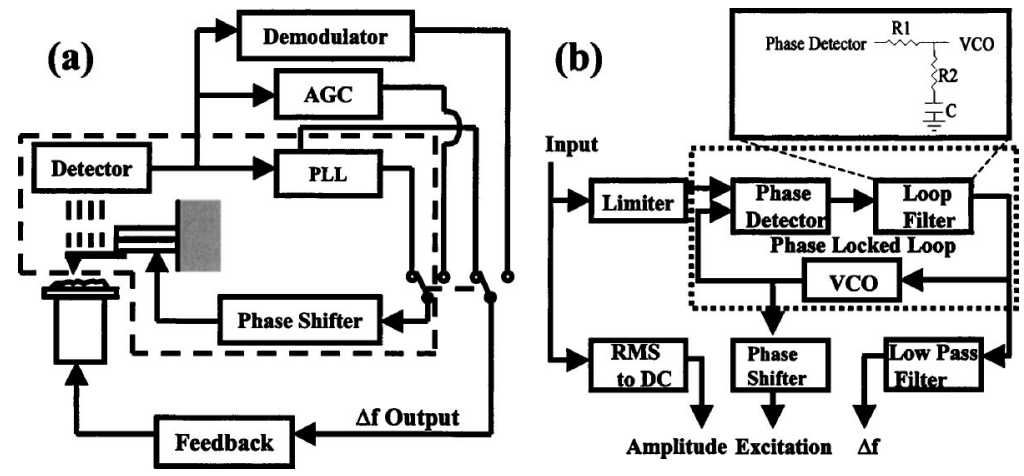

FIG. 1. (a) Schematic diagram of a phase locked oscillator (PLO) and a conventional direct resonance oscillator (DRO). The PLO is made of a detector, a phase locked loop (PLL) and a phase shifter while the DRO consists of an automatic gain control (AGC) and a phase shifter. In situ comparison is made between the PLO and DRO by toggling multiple switches. (b) Detailed schematic block diagram of the electronic circuit in the PLO. A limiter clips the response signal from detector to $\pm 0.6 \mathrm{~V}$ to reduce the spur noise due to the change of input amplitude. The output of the phase detector is time-averaged by a loop filter, PLL, to eliminate high frequency ripple. It is then used as the input signal of a voltage controlled oscillator (VCO). The resultant sensitivity of $\Delta f$ output is designed to be $5 \mathrm{mV} / \mathrm{Hz}$ at $f_{c}=270 \mathrm{kHz}$. Positive feedback is attained with a phase shifter by making an in-phase relationship between the response of cantilever vibration and excitation signal from VCO. A rms to dc converter circuit (AD536 Analog Device) measures the root mean square amplitude. (Inset) A loop filter in second-order which consists of output resistances $R_{1}$ and $R_{2}$ and capacitance $C$.

sweeps with a variable resistor between $200 \mathrm{kHz}$ and 350 $\mathrm{kHz}$ until $f_{c}$ equals the resonance of the cantilever, $f_{0}{ }^{12}$ At $f_{c}=f_{0}$, the lock-in state is also acquired between the excite phase, $\theta_{\text {excite }}$, and the response phase, $\theta_{\text {response }}{ }^{13,14}$

One can adjust the bandwidth of the PLL by changing the output resistances $R_{1}$ and $R_{2}$ and the capacitance $C$ in the loop filter as shown in the inset of Fig. 1(b). The loop response function of PLL, $H(j \omega)$, on modulation frequency $\omega$ between $\theta_{\text {excite }}$ and $\theta_{\text {response }}$ can be written as ${ }^{15}$

$$
H(j \omega)=\frac{j \omega \omega_{n}\left(2 \zeta-\omega_{n} / K_{o} K_{d}\right)+\omega_{n}^{2}}{-\omega^{2}+2 j \zeta \omega_{n} \omega+\omega_{n}^{2}},
$$

where the natural frequency and the damping constant are given, respectively, by

$$
\begin{aligned}
& \omega_{n}=\sqrt{\frac{K_{o} K_{d}}{R_{1} C+R_{2} C}}, \\
& \zeta=\frac{1}{2} \sqrt{\frac{K_{o} K_{d}}{R_{1} C+R_{2} C}}\left(R_{2} C+\frac{1}{K_{o} K_{d}}\right)
\end{aligned}
$$

for the sensitivities of the phase detector and the VCO, $K_{d}$ $=0.68(\mathrm{~V} / \mathrm{rad})$ and $K_{o}=4.1 f_{c}(\mathrm{rad} / \mathrm{s} / \mathrm{V})$ with supply voltage $V=12 \mathrm{~V}$, respectively. ${ }^{12}$ The natural frequency $\omega_{n}$ $=2 \pi \cdot 360 \mathrm{rad} / \mathrm{s}$ and damping constant $\zeta=1.0$ are selected so that the phase error, $\theta_{\text {excite }}-\theta_{\text {response }}$, can be less than 1 radian or $57^{\circ}$, leaving a $33^{\circ}$ margin for noise. With loop gain $K_{o} K_{d}=33.6 f_{c} / V(1 / \mathrm{s}), \quad f_{c}=270 \mathrm{kHz}, \quad$ and $V=14 \mathrm{~V}$ for LM $565,{ }^{12}$ time constants $R_{1} C=1.96 \times 10^{-2} \mathrm{~s}$ and $R_{2} C=4$ $\times 10^{-4} \mathrm{~s}$ can be found from Eqs. (2) and (3). For a given output resistance $R_{1}=3.6 \mathrm{k} \Omega$, I find $C_{1}=5.44 \mu \mathrm{F}$ and $R_{2}$ $=73.5 \Omega$.

I measure the background noise, the temporal response, and frequency shift $(\Delta f)$ versus distance curve to check the performance of PLO over conventional DRO. An $n+$ doped $\mathrm{Si}$ cantilever with resistivity $0.01-0.02 \Omega \mathrm{cm}$ (Nanosensor) is employed for these measurements. Silicon oxide on the surface of tip is removed by sputtering with $1 \mathrm{keV} \mathrm{Ar+} \mathrm{for} 3 \mathrm{~min}$ to get a clean Si surface. Stiff silicon cantilevers with resonance frequency about $f_{0}=270 \mathrm{kHz}$ and spring constant $k=21-78 \mathrm{~N} / \mathrm{m}$ are used as force sensors to prevent the cantilever from having "snap to contact" with the surface. Oscillation peak to peak amplitude of the cantilever is about $30 \mathrm{~nm}$ under UHV environment with a base pressure $\sim 1 \times 10^{-10}$ Torr.

First, the background noise with PLO far away from the surface is lower than that of conventional DRO by a factor $\sim 3$ as found in the top and bottom of Fig. 2(a). This result is consistent with the generally known noise reduction of a PLL circuit, compared to the conventional demodulator. ${ }^{15}$

The bandwidth of PLO should be high enough to get a practical scanning speed close to the sample surface during
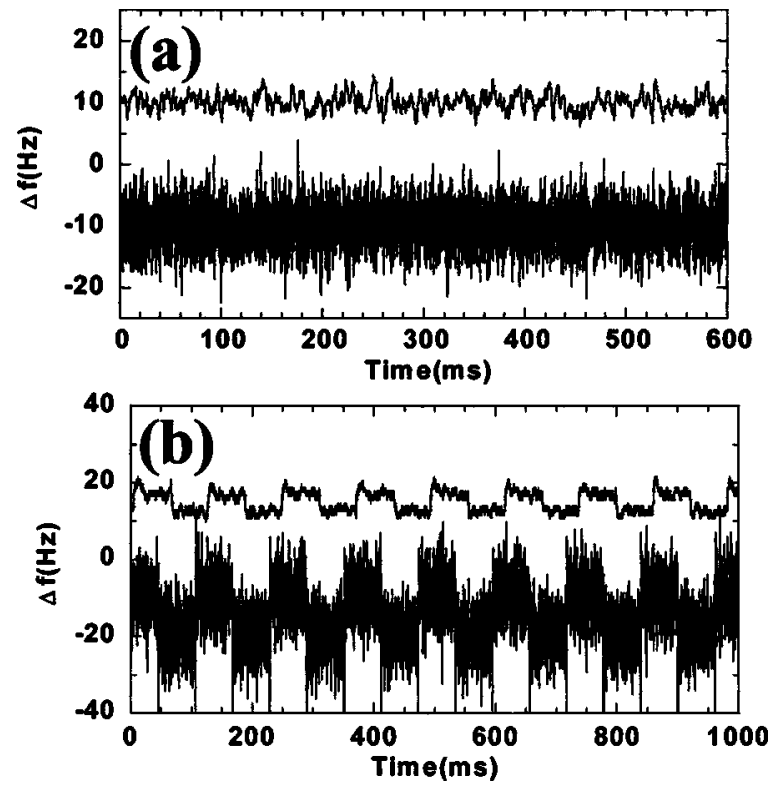

FIG. 2. (a) Background noises of (top) the PLO system and (bottom) the DRO system with the root mean square (rms) values, $1.32 \mathrm{~Hz}$ and $3.46 \mathrm{~Hz}$, respectively. The acquisition time and the sampling rate are $0.7 \mathrm{~s}$ and $6.76 \mu$ s/point, respectively, for both measurements. (b) Temporal responses of (top) the PLO system and (bottom) the DRO system using electrostatic force modulation between tip and sample, measured with driving frequency $4 \mathrm{~Hz}$. In the PLO system, the measurement settings are natural frequency $\omega_{n}=2 \pi \cdot 360 \mathrm{rad} / \mathrm{s}$ and damping constant $\zeta=1.0$. 


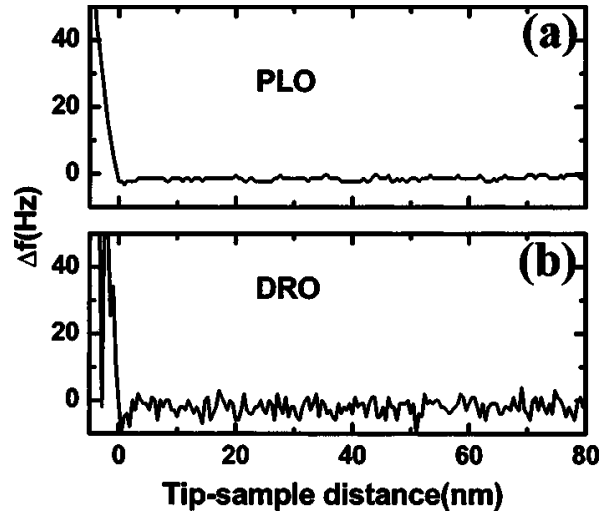

FIG. 3. Frequency shift versus distance curves taken on a clean VC (100) surface with noncontact mode AFM using (a) PLO and (b) DRO.

imaging and force-profile measurement. In order to look at time dependent response of feedback in PLO, I created a time-dependent force field varying close to the sample surface using an attractive electrostatic force modulation. The capacitive force $F_{c}$ can be written down as

$$
F_{c}(z)=\frac{1}{2} \frac{\partial C(z)}{\partial z} V^{2}
$$

where $C(z)$ is the capacitance between tip and sample and is a function of the instaneous tip-sample distance $z$. Due to the square law in Eq. (4), a second harmonic force is generated with modulation frequency $2 \Omega$ for a given driving frequency $\Omega$. A square wave is applied between tip and sample directly, with an amplitude of $10 \mathrm{~V}$ and a driving frequency of $4 \mathrm{~Hz}$ using the VCO of a function generator. ${ }^{16}$

The top and bottom of Fig. 2(b) show the output signals measured with PLO and DRO, respectively. These signals are taken on a conductive VC(100) single crystal. The surface is cleaned with Ar ion sputter/ anneal cycles in ultrahigh vacuum. ${ }^{17}$ Both response signals show the square function with frequency $8 \mathrm{~Hz}$ with some finite response time. The measured bandwidth of the PLO is found to be over $340 \mathrm{~Hz}$ when the driving frequency $\Omega$ is swept between tip and sample. This results suggests that the PLO enables one to get an image within a practical time scale (5-10 $\mathrm{min}$ ).

In addition to the background noise and the bandwidth, I measured frequency shift $(\Delta f)$ as the tip approaches a clean $\mathrm{VC}(100)$ surface to understand the stability of both oscillators against external disturbances. Figures 3(a) and 3(b) show a typical frequency shift $(\Delta f)$ versus distance curves for PLO and DRO, respectively. In the repulsive force regime, the $\Delta f$ signal tracks the interaction very well for PLO but it jitters for DRO. This result indicates that PLO is much more stable than DRO against external disturbances. The result suggests that the phase coherent effect of PLO should enhance the stability of imaging as well as reduce the noise level. Since the roots of the denominator in Eq. (1) have only positive imaginary parts, the phase relation between $\theta_{\text {excite }}$ and $\theta_{\text {response }}$ will be unconditionally stable for all gains and frequencies. ${ }^{15}$ This is believed to be the reason why stable, reproducible atomic images have been obtained since the employment of PLO in NC AFM systems. ${ }^{18,19}$ This is also consistent with the observation by Reichling et al. of a resolution difference in imaging $\mathrm{CaF}_{2}(111)$ using PLO and DRO systems. ${ }^{20}$

The author wishes to thank Dr. Scott Perry for support of the work, Dr. Hyun I. Kim for assistance with construction of the electronics described, and Dr. Antonio Oliver for his careful reading of the manuscript and correcting errors during preparation.

${ }^{1}$ F. J. Giessibl, Science 267, 68 (1995).

${ }^{2}$ S. Kitamura and M. Iwatsuki, Jpn. J. Appl. Phys., Part 2 34, L145 (1995).

${ }^{3}$ Y. Sugawara, M. Ohta, H. Ueyama, and S. Morita, Science 270, 1646 (1995).

${ }^{4}$ R. Lüthi, E. Meyer, M. Bammerlin, A. Baratoff, T. Lehmann, L. Howald, Ch. Gerber, and H.-J. Güntherodt, Z. Phys. B: Condens. Matter 100, 165 (1996).

${ }^{5}$ T. R. Albrecht, P. Grütter, D. Horne, and D. Rugar, J. Appl. Phys. 69, 668 (1991).

${ }^{6}$ Ch. Loppacher, M. Bammerlin, M. Guggisberg, F. Battiston, R. Bennewitz, S. Rast, A. Baratoff, E. Meyer, and H.-J. Güntherodt, Appl. Surf. Sci. 140, 287 (1999).

${ }^{7}$ P. Grütter, Y. Liu, P. LeBlanc, and U. Dürig, Appl. Phys. Lett. 71, 279 (1997).

${ }^{8}$ U. Dürig, H. R. Steinauer, and N. Blanc, J. Appl. Phys. 82, 3641 (1997).

${ }^{9}$ U. Dürig, O. Züger, and A. Stalder, J. Appl. Phys. 72, 1778 (1992).

${ }^{10}$ W. A. Atia and C. C. Davis, Appl. Phys. Lett. 70, 405 (1997).

${ }^{11}$ A. D. L. Humphris, J. Tamayo, and M. J. Miles, Langmuir 16, 7891 (2000).

${ }^{12}$ Data sheet of LM565 (National Semiconductor).

${ }^{13}$ W. H. J. Rensen, N. F. van Hulst, A. G. T. Ruiter, and P. E. West, Appl. Phys. Lett. 75, 1640 (1999).

${ }^{14}$ H. Edwards, L. Taylor, W. Duncan, and A. J. Melmed, J. Appl. Phys. 82, 980 (1997).

${ }^{15}$ F. M. Gardner, Phaselock Techniques (Wiley, New York, 1966).

${ }^{16}$ TEMA:2 MHz function generator.

${ }^{17}$ B. I. Kim, S. Lee, R. L. Guenard, L. C. Fernandez-Torres, S. S. Perry, P. Frantz, and S. V. Didziulis, Surf. Sci. 481, 185 (2001).

${ }^{18}$ Ch. Loppacher, M. Bammerlin, F. Battiston, M. Guggisberg, D. Müller, H. R. Hidber, R. Lüthi, E. Meyer, and H.-J. Güntherodt, Appl. Phys. A: Mater. Sci. Process. 66, S215 (1998).

${ }^{19}$ T. Uchihashi, Y. Sugawara, T. Tsukamoto, M. Ohta, and S. Morita, Phys. Rev. B 56, 9834 (1997).

${ }^{20}$ A. S. Foster, C. Barth, A. L. Shluger, and M. Reichling, Phys. Rev. Lett. 86, 2373 (2001). 